\title{
Cognitive Behavioural Analysis System of Psychotherapy for Treatment- Resistant Depression: Adaptation to a Group Modality
}

\author{
Liliane Sayegh,' Kenneth D. Locke, ${ }^{2}$ Daniele Pistilli, ${ }^{\prime}$ J. Kim Penberthy, ${ }^{3}$ \\ Eduardo Chachamovich,' James P. McCullough Jr., ${ }^{4}$ and Gustavo Turecki ${ }^{5}$ \\ ${ }^{\prime}$ Douglas Mental Health University Institute, Canada \\ ${ }^{2}$ Department of Psychology and Communication Studies, University of Idaho, USA \\ ${ }^{3}$ Department of Psychiatry \& Neurobehavioral Sciences, University of Virginia, USA \\ ${ }^{4}$ Department of Psychology, Virginia Commonwealth University, USA \\ ${ }^{5}$ Department of Psychiatry, McGill University, Canada
}

\begin{abstract}
Studies researching psychotherapeutic interventions for treatment-resistant depression (TRD) are quite new to the field. The Cognitive Behavioural Analysis System of Psychotherapy (CBASP) is the only model developed specifically to treat the chronically depressed patient. While empirical evidence indicates that CBASP is an effective treatment for chronic depression, little is known about its adaptation to a group modality. Treating these patients in a group approach would have the added benefits of being cost-effective and providing in vivo previously avoided interpersonal situations for practising social skills and role-plays. This single arm study asks whether CBASP adapted to a group modality can be effective. All patients received 12 CBASP group therapy sessions with two to four individual preparatory sessions before the group. Our results suggest that CBASP group treatment demonstrated positive effects on patient outcomes. Specifically, patients showed significant decreases in symptoms of depression and the use of emotion-oriented coping, as well as increases in overall social adjustment and interpersonal self-efficacy when compared to pretreatment levels. However, patients did not achieve normative levels in these areas by the end of treatment. These pilot results are encouraging and support further study of the effectiveness of CBASP group treatment with a control group.
\end{abstract}

Keywords: TRD, depression, cognitive, group, therapy

Little is known about psychotherapeutic interventions for treatment-resistant depression (TRD) and studies researching this increasing clinical reality are quite new to the field (Rush, Thase, \& Dube, 2003). The World Health Organization (Greden, 2001) has predicted that unipolar depression alone will be the second most significant contributor to disability by the year 2020. Stewart, Ricci, Chee, Hahn, and Morganstein (2003) estimated the cost to employers of United States workers with depression to be $\$ 44.01$ billion per year in lost productive time, which is about $\$ 31$ billion more per year compared with an expected cost for workers without depression. The epidemiology of treatment-resistant depression is still not well known and varies

Address for correspondence: Liliane Sayegh, Psychologist, Depressive Disorders Program, Douglas Mental Health University Institute, 6875 Boul. LaSalle Montréal, Qc, Canada, H4H 13R.

Email: lilian.sayegh@douglas.mcgill.ca 
according to the population studied (primary care, general population or secondary care) and the definition used.

Only about $20 \%$ to $40 \%$ of patients receiving their first treatment for a major depressive episode are expected to achieve a relatively asymptomatic state (Sackeim, 2001). Even then, there is often a lag until a full recovery of social and occupational functioning is achieved (Fava, Ruini, \& Belaise, 2007; Rush et al., 2004; Sackeim, 2001). The Sequenced Treatment Alternatives to Relieve Depression (STAR*D) study estimated that 33\% of patients treated for up to four courses of treatment, did not remit (Rush et al., 2006). Other authors report a 50\% response (i.e., have a $\geq 50 \%$ reduction in baseline symptom severity) rate for outpatients with nonpsychotic major depressive disorder (MDD) initially treated with either a time-limited depressiontargeted psychotherapy or a single antidepressant medication (Rush et al., 2004). According to the NIMH Collaborative Depressive Study, about 20\% of patients with MDD will develop a chronic course of the illness (Keller et al., 1984).

There are very few published studies demonstrating the effectiveness of individual or group psychotherapy for TRD. In fact, most outcome studies of psychotherapy describe this treatment-resistant population in their exclusion criteria. Some studies have found cognitive therapy to be as effective as medication in the treatment of moderate to severe major depression (DeRubeis, Gelfand, Tang, \& Simons, 1999; DeRubeis et al., 2005; Hollon et al., 2005). Others also found similar response and remission rates for patients receiving cognitive psychotherapy alone or in combination with citalopram, compared to patients assigned to medication-only second-step strategies, for more severe major depression (Thase et al., 2007). Based upon current research, the best approach to use for treatment-resistant depression appears to be a combination of psychotherapy and pharmacotherapy with some plan for treatment continuation for aftercare (Keller, 2001; Michalak \& Lam, 2002; Paykel et al., 1999; Scott, Palmer, Paykel, Teasdale, \& Hayhurst, 2003; Thase, Friedman, \& Howland, 2001; Whisman, Miller, Norman, \& Keitner, 1991).

The Cognitive Behavioural Analysis System of Psychotherapy (CBASP), developed by McCullough $(2000,2006)$, is the only psychotherapy model developed specifically to treat the chronically depressed patient. This model is based on contemporary learning theory with its primary goals being: (1) to connect the patient perceptually to others (the environment) so that others can begin to inform/influence the behaviour of the patient in positive ways (CBASP is based on a person by environment causal determinant model of behaviour); and (2) to acquire stimulus learning (through the therapeutic and other more adaptive relationships) and response learning (acquire more adaptive coping behaviours to reduce interpersonal avoidance and increase positive reinforcements; McCullough, 2008).

Keller et al. (2000) mounted a long-term, multi-site clinical trial showing the bestyet response rates for chronic depression when CBASP and nefazodone are combined. The overall rates of response for the entire sample at the last follow-up visit were $73 \%$ for the combined-treatment group and $48 \%$ for each of the other monotherapies. Among those who completed the study, remission rates were $24 \%$ for the psychotherapy group, $22 \%$ for the nefazodone group and $42 \%$ for the combined-treatment group. The combined-treatment group also had a lower rate of deterioration at the end of a 16-week continuation phase of the treatment (Kocsis et al., 2003) than the other two monotherapies. Finally, maintenance CBASP therapy, beyond 6 months after recovery, was also found to help prevent relapse in depression, compared to an assessment-only follow-up (Klein et al., 2004). 
The Keller et al. (2000) study also demonstrated for the first time that improvements in psychosocial functioning are greater for the combined nefazodone and CBASP treatment group than for either monotherapy group alone, and these improvements appear to be related to the social skills learned in therapy (Hirschfeld et al., 2002). For all three groups, psychosocial functioning improved more slowly than depressive symptoms at the end of the 12-week acute phase treatment period.

Although CBASP has demonstrated effectiveness in treating chronic depression (Keller et al., 2000), strong early results have been tempered by more recent results (Kocsis et al., 2009b) in which it was found that neither CBASP nor Supportive Psychotherapy was more effective than medication alone for chronically depressed treatment resistant patients. The lack of positive findings in that study were hypothesised to be related to the small number of CBASP sessions $(M=12.5)$ and the effect of an aspect of the study design that focused on pharmacological switching and augmentation, which may have had a negative impact on participants' interest in and expectation of, psychotherapy. Kocsis et al. (2009a) reported elsewhere significantly higher remission rates for patients with MDD who received their preferred treatment, psychotherapy or medication monotherapy, rather than the alternate combination treatment (nefazodone and CBASP). Remission rates were even higher for those who preferred and received psychotherapy (CBASP) as a monotherapy than for those who received a combination of CBASP and nefazodone treatment. CBASP has been used more recently to treat chronically depressed pregnant smokers and was found to be more effective than standard treatment at increasing abstinence and decreasing depressive symptoms 6 months post-treatment. Indeed, CBASP was more effective in women with higher levels of baseline depressive symptoms (Cinciripini et al., 2010).

\section{Group Psychotherapy Research for Chronic Depression}

Very few studies have been published using a group approach to treat chronic or treatment-resistant depression and none using a group-CBASP treatment with a comparison group. A meta-analysis of 48 studies evaluating the effectiveness of group psychotherapy for adults with unipolar, nonpsychotic depression revealed that $93.5 \%$ of studies demonstrated that group psychotherapy significantly decreases depressive symptoms following treatment (McDermut, Miller, \& Brown, 2001). The authors point out that these findings cannot be generalised to severe depression as the data collected was on individuals diagnosed with moderate depression.

There is evidence that modified Interpersonal Psychotherapy (Schramm et al., 2008) and Dialectical Behaviour Therapy-based skills training groups (Harley, Sprich, Safren, Jacobo, \& Fava, 2008) for chronically depressed patients are beneficial. Also, Group Cognitive Behavioural Therapy (GCBT) was found to be as effective as individual CBT and more effective than other group modalities, in a review of 34 papers evaluating the effectiveness of GCBT for unipolar depressive disorders (Oei $\&$ Dingle, 2008). All these studies report a greater relief of the depressive symptomatology, while changes in cognitive, behavioural and social functioning lag behind. The more chronically depressed patients tend to show residual symptoms (including irritability, hopelessness and anxiety) after treatment than patients with milder forms of depression and consequently may need a longer course of treatment (Bristow \& Bright, 1995; Harley et al., 2008; Matsunaga et al., 2010; Oei \& Dingle, 2008; Saulsman, Coall, \& Nathan, 2006). 
CBASP has not yet been implemented in a group format to an outpatient population with treatment resistant depression. Treating chronically depressed patients in a group approach would have the added benefits of being cost-effective and providing an interpersonal context in which patients can practice social skills, thus face in vivo previously avoided interpersonal situations. Improving social functioning may help reduce the risk of relapse/recurrence of major depression for this treatment-resistant population (Petersen et al., 2004; Solomon et al., 2004; Vittengl, Clark, \& Jarrett, 2009).

The purpose of this pilot study was to evaluate the efficacy of CBASP provided in a group format to outpatients with treatment resistant major depression and to examine the impact of the intervention on their depressive symptoms and psychosocial functioning. We hypothesised that at the end of treatment with group-CBASP, patients would report improvement in depressive symptoms and psychosocial functioning when compared to their pretreatment levels.

\section{Method}

\section{Participants}

Participants for this study were recruited between 2008 and 2010 from the Depressive Disorders Program (DDP) at the Douglas Mental Health University, Montreal, Quebec Institute. This specialised outpatient clinic serves adults aged 18 to 65 suffering from MDD, and is responsible for servicing a catchment area of more than 500,000 individuals. The participants included 26 women and 18 men $(M$ age $=47.1$ and $46.4, \mathrm{SD}=7.3$ and 9.9 , respectively). Most patients completed high school ( $M$ years education $=14.0, S D=3.0$ ) and were employed at the time this study was conducted $(75 \%)$. However, only $9.1 \%$ of the sample was actively working, whereas $65.9 \%$ were on sick leave and $25.0 \%$ were unemployed. More patients were single or divorced $(54.4 \%)$ than were married $(45.5 \%)$. All participants had unipolar TRD as defined by (1) a diagnosis of MDD according to a SCID-I interview (First, Gibbon, Spitzer, \& Williams, 1996) using DSM-IV criteria (American Psychiatric Association, 2000) and (2) having failed to respond to at least two adequate trials of antidepressant medications. All patients had an MDD diagnosis of moderate to severe intensity $($ M HAM-D score $=28.9, \mathrm{SD}=6.9) ; 32 \%$ had a recurrent MDD. About $70 \%$ had an average of three past episodes of depression and the average duration of the current episode was almost 3 years (31 months). In addition, about $34 \%$ of patients had a comorbid anxiety disorder while the mean score for anxiety for all patients was in the moderate range (M HAM-A score $=23.4, S D=8.0$ ). We investigated the presence of personality disorders by means of the SCID-II interview (First, Spitzer, Gibbon, \& Williams, 1996). Only five patients received a diagnosis of personality disorder.

Exclusion criteria for participation in the study were: bipolar disorders, cyclothymic disorder, psychosis, and a primary diagnosis other than MDD. Also excluded were patients with a high risk for suicide as these patients required individual therapy. All patients received routine clinical management appointments examining the symptomatology and tolerance of medication and the use of standard antidepressants. All participants in the study gave their informed consent and this study was approved by the ethics and research committee of the Institute. Although patients were often reluctant to participate in group therapy, they all accepted to try the group, and only two patients discontinued after one and two sessions due to their suicidal ideation, which was more disturbing for them within the group. 


\section{Procedure}

This is a single arm study, thus all patients received group-CBASP. Patients were admitted to the group on a consecutive basis, as they entered the program, were informed of the treatment and consented to participate provided they did not meet any exclusion criteria. Patients received two to four individual sessions before beginning their participation in group therapy. The purpose of these sessions was to discuss the chronic nature of the patients' depression and to complete an exercise called the Significant Other History, an essential step in the CBASP model. The patients also completed the Interpersonal Questionnaire (developed by McCullough) which was used to help identify the Interpersonal Domain that appears to be most problematic for the patient. Following these individual sessions, participants began group therapy at the outpatient clinic for a 2-hour session each week for 12 to 13 weeks. Each group had a maximum of six patients with an average group size of five patients. Patients have the option of doing a second or third group after they finish the first set of 12 group sessions, although the data reported here is only at the end of their first group. A total of 12 CBASP-groups were given during the 3-year period in which this pilot study was carried out. As mentioned, some of the patients in these groups were participating for the second or third time. The group therapist (first author) who carried out all the groups in French or in English is a CBASP-certified therapist and trainer as well as being a senior therapist with over 20 years experience in psychiatry and 6 years experience in a specialised program for refractory depression. One cotherapist, who is a trained $\mathrm{PhD}$ psychology resident, participated in each group with the first author, who also trained them.

\section{Group-CBASP methodology}

CBASP is a model based on contemporary learning theory (McCullough, 2008). As such, the group-CBASP manual that has been developed at the Depressive Disorders Program of the Institute uses the basic learning paradigm of the model.

The group sessions begin with brief personal introductions of each group member followed by an explanation of the outline of the group sessions. Then, everyone completes a brief, standardised, self-report questionnaire on symptoms of depression in the past month. This helps the new and often uncomfortable members talk about their depressive symptoms while maintaining some degree of privacy regarding issues that they would rather not reveal at that time. A discussion follows of the particular manifestation of the depressive symptoms for each member. Following this first group discussion, the group leaders explain how a diagnosis of major depressive illness is obtained and discuss how this differs from dysthymia and chronic depression. With the help of some graphs, definitions are provided of what is a relapse and a recurrence and the importance of compliance and maintenance of long-term pharmacotherapy for recurrent depressions. Another group discussion is held around the particular course of each member's depressive illness. For all the participants, having received a diagnosis of the more severe or chronic form of major depression, hearing other members share their experiences often helps them feel reassured that they are not alone in experiencing the symptoms that they have. A discussion regarding their experiences with medication ensues. This first group session ends with a suggestion to members that they chart their mood over the course of the next month using the mood chart. Then a homework assignment is given asking the members to chart on an activity schedule their typical activities in the next week, including times at which 
they wake up and go to sleep, times at which they take their meals, go out for a walk and even do their personal hygiene. They are also instructed to include any social interactions that they may have during the week, even if these are telephone calls with a friend.

In the second group session, the activity charts are reviewed and a discussion is held on the actual lifestyle of each group member in their present state of health. The group leaders identify aspects of participants' daily routine that may be problematic, particularly with regards to the frequently mentioned isolation that results from their avoidance of contact with just about anyone. The therapists then present a behavioural activation module on healthy living, which includes a balanced diet, physical activity, good sleeping habits, attention to personal hygiene, ways to stimulate one's cognitive functioning, and attention to one's environment. The idea of reintroducing positive reinforcements and pleasure experiences, which have been dramatically reduced since the onset of the depression, is discussed. Members are encouraged to choose one area of their personal lifestyle that they need to make changes in, and to choose one social activity that would represent a challenge for them but that is also a preferred activity. These activities may be in the area of physical exercise, eating or sleep habits, or in improving personal hygiene. The concept of graded task assignments is explained and positive reinforcement from discussing these challenges with peers in the group who understand them is very supportive for patients. The members are asked to identify a time in the week when they will begin to put into practice these activities. Every week until the end of the group sessions, members discuss at the beginning of each session how they were able to carry out the challenge for the past week and then identify a new level for this challenge for the following week, or perhaps identify a new challenge. This summarises the behavioural activation module that is integrated into the CBASP group therapy program.

The third group session introduces the CBASP model with a discussion on the cycle of hopelessness and global thinking that generates helplessness and defeatism, which in turn results in feeling misunderstood by others and in avoidance of others. The longstanding isolation brings about the perception, described very well by McCullough (2000), on the part of chronically depressed individuals that they have no effect on others in their environment and that others' feedback has little if any impact on them, nor informs their own behaviour. This results in the inability of these individuals to identify interpersonal motives for their behaviours that are intrinsic and self-determined. They do not understand or identify the consequences of their own behaviours on others, or vice versa. The interaction between the person and the environment is severed, as described by McCullough, as these individuals begin to feel that they have lost control over their lives.

The therapists introduce the exercise of the Situational Analysis as the way in which the group members can break the vicious cycle of chronic depression and learn to regain control over their lives by learning how to solve one interpersonal problem at a time. The exercise is explained using one member's example of an interpersonal interaction.

During the remainder of the group therapy, members are asked to bring each week an example of an interpersonal interaction that they will have attempted to write down on the exercise sheet, following the five steps. During each group session, each member has an opportunity to share their interpersonal challenge of the week, and all remaining members participate in developing the four steps, following the description of one person's situation. 
The Situational Analyses also give the therapists an opportunity to identify any social skills deficits each member may have and encourage them to learn interpersonal skills through role plays and in-vivo exposure with other group members. In addition, the technique of Interpersonal Discrimination is used within the group session whenever a group member has been confronted with an Interpersonal Domain that had been identified as difficult for them during the individual sessions prior to the beginning of group therapy. This provides an opportunity to use an operant conditioning paradigm to show patients how they can change their attitudes and behaviours with reinforcements from the group.

The patients' improvements in empathy are seen in their greater capacity to give each other advice during Situational Analysis exercises over the course of group therapy.

\section{Assessment Measures}

All patients underwent a comprehensive psychiatric evaluation at baseline, including the SCID-I and SCID-II, as well as the Hamilton (1967) scales for depression and anxiety (HAM-D and HAM-A). In addition, they completed the Beck Depression Inventory BDI (Beck, Ward, Mendelson, Mock, \& Erbaugh, 1961); Social Adjustment Scale- Self-Report SAS-SR (Weissman, 1999); the Coping Inventory for Stressful Situations CISS (Endler \& Parker, 1999); the Circumplex Scales of Interpersonal Values CSIV (Locke, 2000); Circumplex Scales of Interpersonal Efficacy CSIE (Locke $\&$ Sadler, 2007); and the Inventory of Interpersonal Problems IIP-32 (Horowitz, Alden, Wiggins, \& Pincus, 2000), before the first group and post-treatment after the last group ended.

\section{Statistical Methods}

We conducted a mixed analysis of variance on each outcome, with pretreatment and post-treatment scores as a within-subjects factor, and gender as a between-subjects factor. The Statistical Package for Social Sciences (SPSS) version 18 was used for the analyses. The default Type I error level was .05 (2-tailed). The outcome variables were the BDI, SAS, IIP, CSIV, and CSIE full-scale scores, and the CISS Task-Oriented, Emotion-Oriented, and Avoidance-Oriented subscale scores.

\section{Results}

All patients who agreed to participate in group therapy completed the 12 sessions. The result revealed three significant gender effects: Males scored lower than females on the BDI, Ms = 23.1 and 30.8, SEs = 2.0 and 2.1, F(1,42)=6.4, and higher than females on the CISS Avoidance-Oriented coping scale, Ms $=47.5$ and 40.5, SDs = 1.6 and 1.3, F(1,42) = 10.1, and the CSIE, Ms = 5.9 and 5.2, SEs $=0.2, F(1,42)=$ 4.6 , ps $<.05$. Gender did not moderate effects of treatment (all $F s<1.5$, ps $>.25$ ).

Table 1 shows the effects of treatment. Self-reported depression symptoms (BDI) decreased from a severe to a moderate level. Overall social adjustment (as assessed by the SAS-SR) also improved. There was a small decrease in emotion-oriented coping, which is a maladaptive problem-solving strategy that aims to reduce stress by getting angry, becoming tense, blaming oneself, or through self-preoccupation and fantasising (Endler \& Parker, 1999) page 33). Finally, there was a marginally significant increase in overall self-efficacy for interpersonal actions (as assessed by the CSIE). 


\section{TABLE 1}

Effects of Treatment on Depression, Coping, and Social and Interpersonal Functioning

\begin{tabular}{|c|c|c|c|c|c|}
\hline & \multicolumn{2}{|c|}{ Pretreatment } & \multicolumn{2}{|c|}{ Post-treatment } & \multirow[b]{2}{*}{$F(1,42)$} \\
\hline & $M$ & (SE) & $M$ & $S E$ & \\
\hline $\mathrm{BDI}$ & 31.1 & $(1.7)$ & 24.2 & (1.8) & $21.1 * *$ \\
\hline SAS-SR & 81.3 & (1.9) & 75.4 & (1.9) & $10.7 * *$ \\
\hline IIP & 70.6 & $(1.1)$ & 69.0 & (1.3) & 0.8 \\
\hline CSIE & 5.4 & $(0.2)$ & 5.7 & $(0.2)$ & $3.1 \dagger$ \\
\hline CSIV & 2.0 & $(0.1)$ & 1.9 & $(0.1)$ & 1.5 \\
\hline CISS : Task-Oriented & 34.9 & (1.3) & 35.6 & (1.3) & 0.2 \\
\hline CISS : Emotion-Oriented & 61.7 & $(1.1)$ & 59.1 & (1.4) & $3.9 *$ \\
\hline CISS : Avoidance-Oriented & 42.9 & (1.3) & 43.9 & (1.4) & 0.4 \\
\hline
\end{tabular}

Note: CSIE values are mean ratings on 0-to-10 scales. CSIV values are mean ratings on 0-to-4 scales. SAS, IIP, and CISS values are T-scores.

$\dagger p \leq .1{ }^{*} p \leq .05 .{ }^{*} p \leq .005$.

The CSIE contains scales that measure interpersonal self-efficacy associated with each region of the interpersonal circumplex. To better understand the increase in self-efficacy, we conducted a mixed analysis of variance on each self-efficacy within each quadrant of the circumplex - that is, self-efficacy for agentic behaviours (e.g., assertion), unagentic actions (being a follower), communal actions (e.g., helping), and uncommunal actions (e.g., being unfriendly) — with pretreatment and post-treatment scores as a within-subjects factor, and gender as a between-subjects factor. Treatment improved self-efficacy for agentic behaviours (pre- and post-treatment, Ms $=4.2$ and 4.8 , SEs $=0.3, F(1,42)=5.1, p<.05$, but did not change self-efficacy in the other quadrants. In addition, males scored higher than females on self-efficacy for agentic behaviours and lower than females on self-efficacy for unagentic behaviours ( $F$ s $>5$, ps $<.05)$, but gender did not moderate the effects of treatment $(F s<1$, ps $>.5)$.

\section{Discussion}

In this small preliminary study utilising CBASP in a group format with treatmentresistant severely depressed patients, CBASP group treatment demonstrated positive effects on patient outcomes as hypothesised. Specifically, at the end of treatment, patients showed significant decreases in self-reported symptoms of depression and in the use of emotion-oriented coping, as well as increases in overall social adjustment and interpersonal self-efficacy when compared to their pretreatment levels. Moreover, the effects on overall depression and adjustment were quite strong. In short, the results

104 of our pilot study were encouraging and support further study of the effectiveness of CBASP group treatment.

At the start of treatment, work roles, social relationships and leisure activities were the most problematic in light of the patients' inability to work and increased withdrawal from all pleasurable activities. Patients did not reach social adjustment levels comparable to the community sample in these areas in spite of the demonstrated improvements. At the end of treatment, patients reported levels of disturbances that were similar to a chronically depressed sample (Weissman, 1999). Scores for 
emotion-oriented coping at the end of group-CBASP were also comparable to a psychiatric normative group for both men and women. Thus, although the patients improved with respect to their pretreatment levels of depression and psychosocial functioning, they did not achieve normative levels in these areas by the end of the 12 weeks of treatment.

These results confirm those found in the REVAMP study (Kocsis et al., 2009b): 12 weeks of CBASP treatment is not sufficient to allow for remission from chronic depression. One can say that the patients in this study also ended group-CBASP with a partial remission, although significant improvements had been achieved in depressive symptoms and in interpersonal functioning by the end of group-CBASP. Patients in the current study received one session per week for a total of 12 group sessions and an average of an additional two pretreatment individual sessions. Patients in the REVAMP study received an average of 16 to 20 sessions within a period of 12 weeks. Evidence suggests that 12 weeks of exposure to CBASP treatment may not be sufficient to ensure that patients with severe levels of depression can acquire the learning they need and generalise it to their environment without further therapeutic help.

Group-CBASP appeared to facilitate the acquisition of interpersonal skills. GroupCBASP helped patients improve their interpersonal self-efficacy in the area of agentic behaviours, which include assertive, self-confident, and independent behaviours. According to social-cognitive learning models, people engage in behaviours that they believe they can successfully enact and that will produce valued outcomes (Bandura, 1997). Therefore, increases in self-efficacy for engaging in various interpersonal behaviours and in the subjective importance of having specific types of interpersonal experiences should facilitate positive interpersonal changes. It is important to note that there were no improvements in self-efficacy scores for the dimension of affiliation on the CSIE. Patients did report that they participated in group therapy to improve their interpersonal functioning and not to simply affiliate with others.

Overall, self-reported interpersonal problems did not decrease by the time the group terminated. It is very probable that the 12 -week duration of this group-CBASP trial was not sufficient to improve interpersonal functioning to a level adequate enough to successfully reintegrate their social roles. We recommend extending the length of treatment to at least 20 sessions in the next phase of this research. Group therapy has been shown to be equivalent or marginally less effective only in the short term, compared to individual therapy (Cuijpers, Van Straten, \& Warmerdam, 2008), but its cost has been found to be less than half that of individual therapy while producing both net health benefits and net cost savings (Vos, Corry, Haby, Carter, \& Andrews, 2005). CBASP lends itself very well to a group modality providing the social laboratory needed to rehearse interpersonal skills learned and obtain positive and negative reinforcements directly from peers. This operant conditioning paradigm is best implemented in a group format, with positive evidence from this pilot study suggesting that patients' interpersonal self-efficacy is developing particularly in the area of agentic domains. It is also more feasible to envisage a cost-effective follow-up with group-CBASP for both continuation and maintenance-phase treatment, particularly for treatment-resistant depression.

Two major limitations of the current study were the absence of a control group and a relatively small sample (especially for males). With a larger sample and the inclusion of a comparison group, we will be better able to distinguish between the effects of the group modality and the effects of the specific skills learned in CBASP. 
With a larger sample, examining the subscales of the instruments used in this study, including both self-report and clinician-rated scales, should also yield a more detailed and nuanced understanding of how CBASP group treatment does (or does not) enhance interpersonal functioning and social adjustment, and how such changes can help promote and sustain the alleviation of other symptoms of major depression.

\section{References}

American Psychiatric Association. (2000). Diagnostic and statistical manual of mental disorders (4th ed., text rev.). Arlington, VA: Author.

Bandura, A. (1997). Self-efficacy: The exercise of control. New York: Freeman.

Beck, A.T., Ward, C.H., Mendelson, M., Mock, J., \& Erbaugh, J. (1961). An inventory for measuring depression. Archives of General Psychiatry, 4, 561-571.

Bristow, M., \& Bright, J. (1995). Group cognitive therapy in chronic depression: Results from two intervention studies. Behavioural and Cognitive Psychotherapy, 23, 373-380.

Cinciripini, P.M., Blalock, J.A., Minnix, J.A., Robinson, J.D., Brown, V.L., Lam, C., .. KaramHage, M. (2010). Effects of an intensive depression-focused intervention for smoking cessation in pregnancy. Journal of Consulting and Clinical Psychology, 78(1), 44-54.

Cuijpers, P., Van Straten, A., \& Warmerdam, L. (2008). Are individual and group treatments equally effective in the treatment of depression in adults? A meta-analysis. European Journal of Psychiatry, 22(1), 38-51.

DeRubeis, R., Gelfand, L., Tang, T., \& Simons, A. (1999). Medications versus cognitive behavior therapy for severely depressed outpatients: Mega-analysis of four randomized comparisons. American Journal of Psychiatry, 156(7), 1007-1013.

DeRubeis, R., Hollon, S., Amsterdam, J., Shelton, R., Young, P., Salomon, R., ... Gallop, R. (2005). Cognitive therapy vs medication in the treatment of moderate to severe depression. Archives of General Psychiatry, 62, 409-416.

Endler, N.S., \& Parker, J.D.A. (1999). Coping Inventory for Stressful Situations (CISS). Toronto: Multi-Health Systems Inc.

Fava, G.A., Ruini, C., \& Belaise, C. (2007). The concept of recovery in major depression. Psychological Medicine, 37(3), 307-317.

First, M.B., Gibbon, M., Spitzer, R.L., \& Williams, J.B.W. (1996a). User's guide for the Structured Clinical Interview for DSM-IV Axis I Disorders - Research version (SCID-I, version 2.0). New York: Biometrics Research Department, New York State Psychiatric Institute.

First, M.B., Spitzer, R.L., Gibbon, M., \& Williams, J.B.W. (1996b). Structured Clinical Interview for DSM-IV Axis II Personality Disorders (SCID-II, version 2.0). New York: Biometrics Research Department, New York State Psychiatric Institute.

Greden, J.F. (2001). The burden of disease for treatment-resistant depression. Journal of Clinical Psychiatry, 62(Suppl 16), 26-31.

Hamilton, M. (1967). Development of a rating scale for primary depressive illness. British Journal of Social \& Clinical Psychology, 6(4), 278-296.

Harley, R., Sprich, S., Safren, S., Jacobo, M., \& Fava, M. (2008). Adaptation of dialectical behavior therapy skills training group for treatment-resistant depression. Journal of Nervous $\mathcal{E}$ Mental Disease, 196(2), 136-143.

Hirschfeld, R.M., Dunner, D.L., Keitner, G., Klein, D.N., Koran, L.M., Kornstein, S.G., . . Keller, M.B. (2002). Does psychosocial functioning improve independent of depressive symptoms? A comparison of nefazodone, psychotherapy, and their combination. Biological Psychiatry, 51(2), $123-133$.

Hollon, S., DeRubeis, R., Shelton, R., Amsterdam, J., Salomon, R., O'Reardon, J., ... Gallop, R. (2005). Prevention of relapse following cognitive therapy vs medications in moderate to severe depression. Archives of General Psychiatry, 62, 417-422. 
Horowitz, L.M., Alden, L.E., Wiggins, J.S., \& Pincus, A.L. (2000). Inventory of Interpersonal Problems (IIP) - Manual. Oxford, UK: The Psychological Corporation a Harcourt Assessment Company.

Keller, M.B. (2001). Long-term treatment of recurrent and chronic depression. Journal of Clinical Psychiatry, 62(Suppl. 24), 3-5.

Keller, M.B., Klerman, G.L., Lavori, P.W., Coryell, W., Endicott, J., \& Taylor, J. (1984). Long-term outcome of episodes of major depression. Journal of the American Medical Association, 252(6), 788-792.

Keller, M.B., McCullough, Jr., J.P., Klein, D.N., Arnow, B., Dunner, D.L., Gelenberg, A.J., ... Zajecka, J. (2000). A comparison of nefazodone, the cognitive behavioral-analysis system of psychotherapy, and their combination for the treatment of chronic depression. New England Journal of Medicine, 342(20), 1462-1470.

Klein, D.N., Santiago, N.J., Vivian, D., Blalock, J.A., Kocsis, J.H., Markowitz, J.C., ... Keller, M.B. (2004). Cognitive-behavioral analysis system of psychotherapy as a maintenance treatment for chronic depression. Journal of Consulting and Clinical Psychology, 72(4), 681688.

Kocsis, J.H., Gelenberg, A.J., Rothbaum, B.O., Klein, D.N., Trivedi, M.H., Manber, R., ... Thase, M.E. (2009b). Cognitive behavioral analysis system of psychotherapy and brief supportive psychotherapy for augmentation of antidepressant nonresponse in chronic depression: the REVAMP Trial. Archives of General Psychiatry, 66(11), 1178-1188.

Kocsis, J.H., Leon, A.C., Markowitz, J.C., Manber, R., Arnow, B., Klein, D. N., \& Thase, M.E. (2009a). Patient preference as a moderator of outcome for chronic forms of major depressive disorder treated with nefazodone, cognitive behavioral analysis system of psychotherapy, or their combination. Journal of Clinical Psychiatry, 70(3), 354-361.

Kocsis, J.H., Rush, A.J., Markowitz, J.C., Borian, F.E., Dunner, D.L., Koran, L.M., . . Keller, M.B. (2003). Continuation treatment of chronic depression: A comparison of nefazodone, cognitive behavioral analysis system of psychotherapy, and their combination. Psychopharmacology Bulletin, 37(4), 73-87.

Locke, K.D. (2000). Circumplex scales of interpersonal values: Reliability, and applicability to interpersonal problems and personality disorders. Journal of Personality Assessment, 75(2), 249267.

Locke, K.D., \& Sadler, P. (2007). Self-efficacy, values, and complementarity in dyadic interactions: Integrating interpersonal and social-cognitive theory. Personality and Social Psychology Bulletin, 33(1), 94-109.

Matsunaga, M., Okamoto, Y., Suzuki, S., Kinoshita, A., Yoshimura, S., Yoshino, A., ... Yamawaki, S. (2010). Psychosocial functioning in patients with treatment-resistant depression after group cognitive behavioral therapy. BMC Psychiatry, 10, 22.

McCullough, Jr., J.P. (2000). Treatment for chronic depression: Cognitive Behavioral Analysis System of Psychotherapy (CBASP). New York: Guilford Press.

McCullough, Jr., J.P. (2006). Treating chronic depression with disciplined personal involvement: CBASP. New York: Springer.

McCullough, Jr., J.P. (2008). The Cognitive Behavioral Analysis System of Psychotherapy: A valueadded strategy for chronic depression. Psychiatric Times, 25(10).

McDermut, W., Miller, I.W., \& Brown, R.A. (2001). The efficacy of group psychotherapy for depression: A meta-analysis and review of the empirical research. Clinical Psychology: Science and Practice, 8(1), 98-116.

Michalak, E.E., \& Lam, R.W. (2002). Breaking the myths: New treatment approaches for chronic depression. Canadian Journal of Psychiatry, 47(7), 635-643.

Oei, T.P., \& Dingle, G. (2008). The effectiveness of group cognitive behaviour therapy for unipolar depressive disorders. Journal of Affective Disorders, 107(1-3), 5-21.

Paykel, E.S., Scott, J., Teasdale, J.D., Johnson, A.L., Garland, A., Moore, R., ... Pope, M. (1999). Prevention of relapse in residual depression by cognitive therapy: A controlled trial. Archives of General Psychiatry, 56(9), 829-835. 
Petersen, T., Papakostas, G.I., Mahal, Y., Guyker, W.M., Beaumont, E.C., Alpert, J.E., .. N Nierenberg, A.A. (2004). Psychosocial functioning in patients with treatment resistant depression. European Psychiatry, 19(4), 196-201.

Rush, A.J., Bernstein, I.H., Trivedi, M.H., Carmody, T.J., Wisniewski, S., Mundt, J.C., ... Fava, M. (2006). An evaluation of the quick inventory of depressive symptomatology and the hamilton rating scale for depression: A sequenced treatment alternatives to relieve depression trial report. Biological Psychiatry, 59(6), 493-501.

Rush, A.J., Fava, M., Wisniewski, S.R., Lavori, P.W., Trivedi, M.H., Sackeim, H.A., .. Niederehe, N. (2004). Sequenced treatment alternatives to relieve depression (STAR*D): rationale and design. Controlled Clinical Trials, 25(1), 119-142.

Rush, A.J., Thase, M.E., \& Dube, S. (2003). Research issues in the study of difficult-to-treat depression. Biological Psychiatry, 53(8), 743-753.

Sackeim, H.A. (2001). The definition and meaning of treatment-resistant depression. Journal of Clinical Psychiatry, 62(Suppl. 16), 10-17.

Saulsman, L.M., Coall, D.A., \& Nathan, P.R. (2006). The association between depressive personality and treatment outcome for depression following a group cognitive-behavioral intervention. Journal of Clinical Psychiatry, 62(9), 1181-1196.

Schramm, E., Schneider, D., Zobel, I., van Calker, D., Dykierek, P., Kech, S., ... Berger, M. (2008). Efficacy of Interpersonal Psychotherapy plus pharmacotherapy in chronically depressed inpatients. Journal of Affective Disorders, 109(1-2), 65-73.

Scott, J., Palmer, S., Paykel, E., Teasdale, J., \& Hayhurst, H. (2003). Use of cognitive therapy for relapse prevention in chronic depression: Cost-effectiveness study. British Journal of Psychiatry, $182,221-227$.

Solomon, D.A., Leon, A.C., Endicott, J., Mueller, T.I., Coryell, W., Shea, M.T., \& Keller, M.B. (2004). Psychosocial impairment and recurrence of major depression. Comprehensive Psychiatry, 45(6), 423-430.

Stewart, W.F., Ricci, J.A., Chee, E., Hahn, S.R., \& Morganstein, D. (2003). Cost of lost productive work time among US workers with depression. JAMA, 289(23), 3135-3144.

Thase, M.E., Friedman, E.S., Biggs, M.M., Wisniewski, S.R., Trivedi, M.H., Luther, J.F., . . Rush, A.J. (2007). Cognitive therapy versus medication in augmentation and switch strategies as second-step treatments: A STAR*D report. American Journal of Psychiatry, 164(5), 739-752.

Thase, M.E., Friedman, E.S., \& Howland, R.H. (2001). Management of treatment-resistant depression: Psychotherapeutic perspectives. Journal of Clinical Psychiatry, 62(Suppl. 18), 18-24.

Vittengl, J.R., Clark, L.A., \& Jarrett, R.B. (2009). Deterioration in psychosocial functioning predicts relapse/recurrence after cognitive therapy for depression. Journal of Affective Disorders, 112(1-3), $135-143$.

Vos, T., Corry, J., Haby, M.M., Carter, R., \& Andrews, G. (2005). Cost-effectiveness of cognitivebehavioural therapy and drug interventions for major depression. Australian and New Zealand Journal of Psychiatry, 39(8), 683-692.

Weissman, M.M. (1999). Social Adjustment Scale Self-Report (SAS-SR): Technical manual. Toronto: Multi-Health Systems Inc.

Whisman, M.A., Miller, I.W., Norman, W.H., \& Keitner, G.I. (1991). Cognitive therapy with depressed inpatients: specific effects on dysfunctional cognitions. Journal of Consulting and Clinical Psychology, 59(2), 282-288. 